\title{
Magnetoresistance of magnetite point contacts and nanoconstrictions
}

\author{
O. Céspedes, ${ }^{\text {a) }}$ E. Clifford, and J. M. D. Coey \\ Physics Department, Trinity College, Dublin 2, Ireland
}

(Received 26 April 2004; accepted 8 December 2004; published online 7 March 2005)

\begin{abstract}
Point contacts of magnetite exhibit large, reversible increases of conductance by a factor of up to 5 in the presence of a small magnetic field $(<10 \mathrm{mT})$, provided the conductance is less than the quantum of conductance $G_{0}(12900 \Omega)^{-1}$. Smaller effects are found in $\left(\mathrm{La}_{0.7} \mathrm{Sr}_{0.3}\right) \mathrm{MnO}_{3}$ and $\mathrm{Co}_{2} \mathrm{Cr}_{0.6} \mathrm{Fe}_{0.4} \mathrm{Al}$. Comparable effects observed in magnetite nanoconstrictions milled using a focused-ion beam in thin films deposited on oxide substrates are only reversible on a time scale of hours. High-resistance nanogaps show evidence of field emission beyond a voltage threshold, which is itself field dependent. The results are discussed in terms of spin-polarized transport, magnetostriction, magnetic dipole strain, and Fowler-Nordheim tunneling. (c) 2005 American Institute of Physics. [DOI: 10.1063/1.1853497]
\end{abstract}

\section{INTRODUCTION}

The question of spin-dependent electron transport across a nanocontact or a nanoconstriction has generated much interest and controversy since the original report of $280 \%$ magnetoresistance in mechanical point contacts by Garcia et al. ${ }^{1}$ Large magnetoresistance values have also been reported in electrodeposited, low-magnetostrictive, permalloy nanocontacts. ${ }^{2}$ Nickel point contacts formed in controlled break junctions ${ }^{3}$ or nanoconstrictions formed in nanopores ${ }^{4}$ usually exhibit a much smaller magnetoresistance of only a few percent. A nanocontact is formed when the surfaces of two crystallites are pressed or grow together, whereas a nanoconstriction is a neck in continuous solid film, which may or may not coincide with a grain boundary. A nanogap arises when there is no direct contact across the junction (Fig. 1). Various electron transport processes can be envisaged in the nanozone between the two sides-diffusive, ballistic, or hopping transport, tunneling or field emission. Furthermore, in magnetically ordered solids, the magnetization may be continuous or discontinuous in the nanozone, depending on whether or not the exchange interactions propagate across it. But even when they do propagate, exchange and magnetic structure may differ from that of the bulk, on account of the preponderance of surface atoms with reduced coordination.

An important observation regarding magnetic nanoconstrictions was made by Bruno, ${ }^{5}$ and subsequently confirmed by numerical simulation. ${ }^{6}$ Domain walls tend to be trapped at or near the nanoconstriction, and the wall width there is of the order $\delta$, the constriction width. It is therefore possible for narrow domain walls to exist in magnetically soft materials. The wall energy is of the order $A \delta$ instead of $(A K)^{1 / 2} S$, where $A$ is the exchange parameter $\left(\approx 10^{-11} \mathrm{~J} \mathrm{~m}^{-1}\right), K$ is the anisotropy constant $\left(10^{2}-10^{5} \mathrm{~J} \mathrm{~m}^{-3}\right)$ and $S$ is the wall area.

A prime concern when measuring magnetic nanocontacts or nanogaps is to distinguish the effects of spin-polarized transport from mechanical effects such as magnetostriction. Any magnetization process which changes the direction of

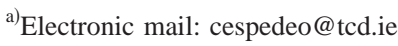

magnetization tends to produce a strain. To first order, the magnetostrictive strain $\varepsilon$ conserves volume; $\varepsilon=\lambda_{s}\left(3 \cos ^{2} \theta\right.$ $-1) / 2$, where $\theta$ is the angle between the measuring direction and the magnetization. The saturation magnetostriction $\lambda_{s}$ is typically $10^{-6}-10^{-4}$. The strain for a crystallite $100 \mu \mathrm{m}$ in size is therefore $0.1-1 \mathrm{~nm}$. It is important to consider magnetostriction when designing and interpreting experiments where tunnel barriers are involved.

Here we focus on magnetoresistance of point contacts, nanoconstrictions, and nanogaps formed from magnetite and other half-metals, where the greatest effects are to be expected. Half-metals exhibit complete spin polarization at the Fermi level when the temperature is far below the Curie point. Most candidate materials are transition-metal oxides or Heusler alloys. ${ }^{7}$

\section{RESULTS}

\section{A. Point contacts}

We have investigated point contacts between two small crystallites of the same material, using a simple piezodevice with vibration isolation. ${ }^{8}$ It is possible to stabilize the resistance of a contact for long enough to be able to measure the $I: V$ characteristic with and without a magnetic field. A typical measurement involves four curves, alternately taken

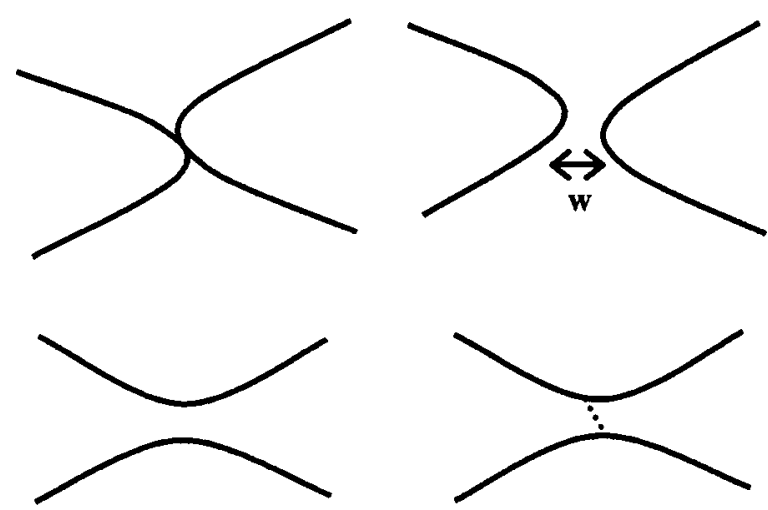

FIG. 1. Sketches of a nanocontact, a nanogap, and a nanoconstriction with or without a grain boundary. 


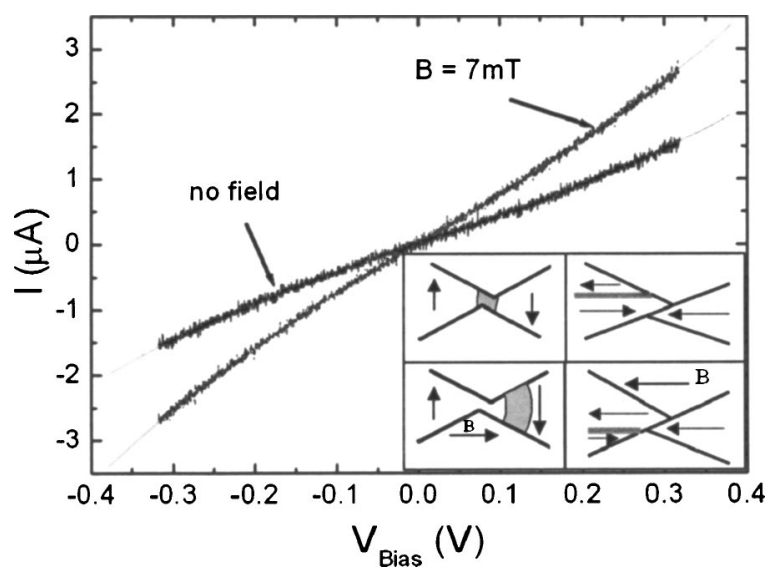

FIG. 2. $I: V$ curves for a magnetite nanocontact, measured with and without an applied field of $7 \mathrm{mT}$. Two possible magnetization processes, which can give rise to a large magnetoresistance, are illustrated in the insets. In one case, a domain wall at the contact is pushed into an adjacent crystallite. In the other case, a wall present in one crystallite is swept across the contact.

without and with an applied field of 8-10 mT. A typical data set is shown in Fig. 2. Some of the data for $\mathrm{Fe}_{3} \mathrm{O}_{4}{ }^{9}$, $\left(\mathrm{La}_{0.7} \mathrm{Sr}_{0.3}\right) \mathrm{MnO}_{3},{ }^{10}$ and $\mathrm{MnBi}^{11}$ have been reported previously, and a summary of the earlier work was published. ${ }^{10}$

The reproducible $I: V$ curves are fitted to the expression

$$
I=G V+C V^{3}
$$

where the conductivity $G$ and the nonlinear coefficient $C$ depend on the field. The same behavior can be parameterized in terms of the height $\phi$ and width $s$ of a rectangular tunnel barrier using the Simmons model; ${ }^{12}$ the conductivity and the nonlinear coefficient can then be expressed as

$$
\begin{aligned}
G & =\frac{3}{2 s}\left(\frac{e}{\hbar}\right)^{2}(2 m \phi)^{1 / 2} \exp \left[-\left(\frac{4 \pi s}{\hbar}\right)(2 m \phi)^{1 / 2}\right], \\
C & =\frac{\pi s m^{3 / 2}}{(2 \phi)^{1 / 2}}\left(\frac{e}{\hbar}\right)^{4} \exp \left[-\left(\frac{4 \pi s}{\hbar}\right)(2 m \phi)^{1 / 2}\right] \\
& =\frac{G s^{2}}{\phi} \times \text { const. }
\end{aligned}
$$

Magnetoresistance is defined as

$$
\Delta \rho / \rho=[G(0)-G(B)] / G(B)=[R(B)-R(0)] / R(0) .
$$

Note that with this definition $\Delta \rho / \rho$ cannot exceed $-100 \%$, and it is negative when the resistance decreases in the applied field, as is usually the case. The "headline" definition with $G(0)$ in the denominator has no lower limit on $\Delta \rho / \rho$.

Figure 3 shows scatter plots of the measurements of magnetoresistance for three different half-metals. A feature of the data on these and other systems ${ }^{11}$ is that there is little magnetoresistance when $G>G_{0}$, but when $G<G_{0}$ the effect can exceed $-80 \%$ (over $500 \%$ increase in conductivity). Here $G_{0}$ is the quantum of conductance $2 e^{2} / h$ $=(12900 \Omega)^{-1}$. A difficulty with these experiments is that there is little control of the contact. When the measurement is repeated, a different contact is established so it is necessary to accumulate a great deal of data and view it statistically. Nevertheless, it is evident from Fig. 3 that $\Delta \rho / \rho$ is

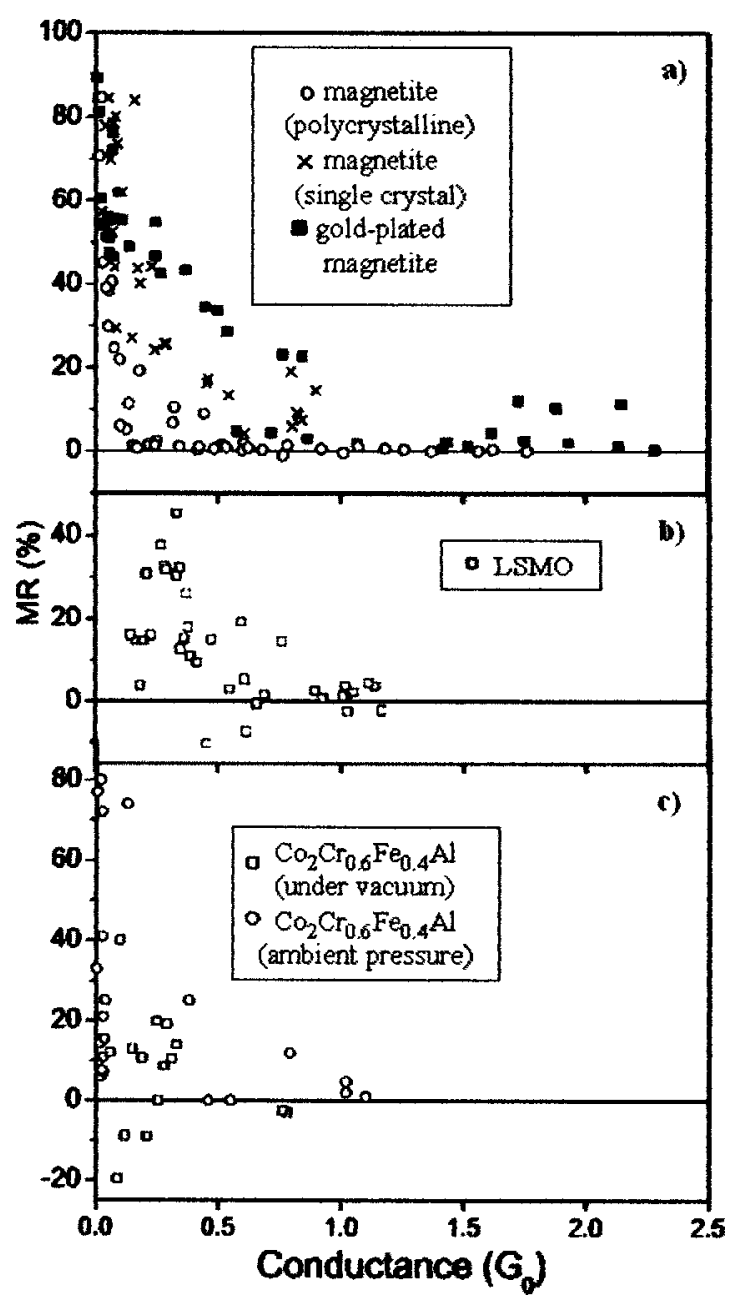

FIG. 3. Plot of the magnetoresistance measured for (a) $115 \mathrm{Fe}_{3} \mathrm{O}_{4=}$ nanocontacts, (b) $37\left(\mathrm{La}_{0.7} \mathrm{Sr}_{0.3}\right) \mathrm{MnO}_{3}$ nanocontacts, and (c) $36 \mathrm{Co}_{2} \mathrm{Cr}_{0.6} \mathrm{Fe}_{0.4} \mathrm{Al}$ nanocontacts. The crosses in (a) are for magnetite crystals which have been coated with a layer of gold.

almost always negative. Only $5 \%$ of all curves show a positive value, and only one contact out of 115 recorded on magnetite. Figure 3(a) includes data on magnetite crystals with a thin surface coating of gold. In this case there is more evidence of magnetoresistance when $G \geqslant G_{0}$ than for the uncoated case.

In fitting the curves to Eq. (1), there is a clear inverse correlation between $C / G$ and $G$; the less conducting the contact, the more nonlinear the $I: V$ curve (Fig. 4). When $G$ $<G_{0}$, there is no direct metallic contact, and some sort of tunnel barrier is formed.

The possibility of magnetostriction, which might be expected to influence the barrier thickness, was investigated in several ways. Some experiments with a point contact between polycrystalline magnetite and a gold electrode established that magnetoresistance of either sign could be observed. In that case, the magnetoresistance was as often positive as negative and the average was zero. In a field rotating at frequency $f=10 \mathrm{~Hz}$, a signal is observed at $2 f$ $=20 \mathrm{~Hz}$, which exceeds the $10 \mathrm{~Hz}$ signal. However, we discount magnetostriction as the major reason for the magnetoresistance of the point contacts shown in Fig. 3 for the following reasons: 


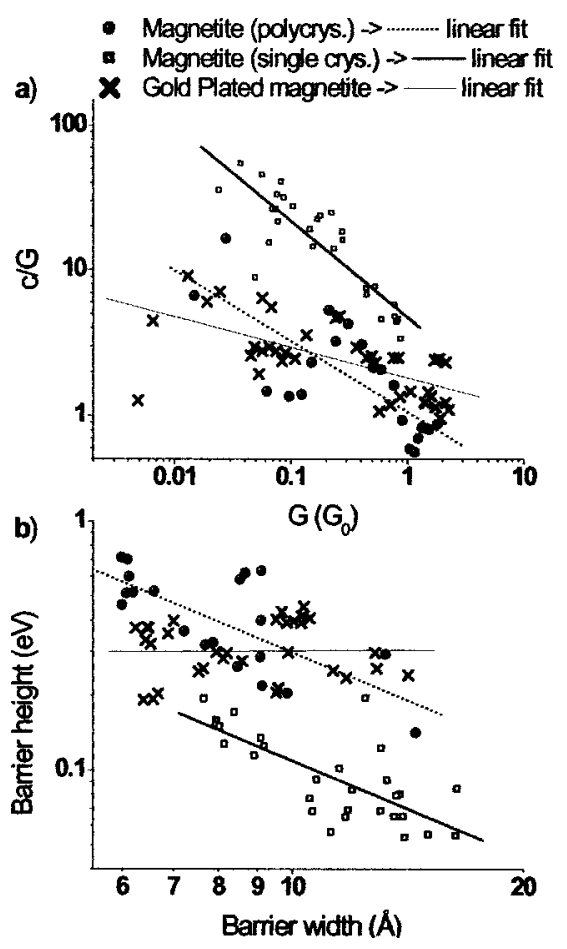

FIG. 4. Plot of $C / G$ vs $G$ (a) and barrier height vs barrier width (b) assuming a cross section of $10 \times 10 \mathrm{~nm}$ for the points in Fig. 3(a). For poly and single crystal magnetite nanocontacts, the barrier height increases with the barrier width with the same slope, but for a certain barrier width, the barrier height is bigger in the case of polycrystalline magnetite than for single crystal. In gold-plated magnetite nanocontacts, the barrier height remains roughly constant for different barrier widths (gap spacing).

- the observed magnetoresistance is almost always negative;

- the signal observed at $2 f$ in a rotating field is much less than the signal at frequency $f$; and

- the initial magnetization process in ferromagnets involves reversible domain wall motion, not a reorientation of the magnetization direction. Hence, the magnetostriction in the small applied fields of $8-10 \mathrm{mT}$ is vanishingly small.

The degree of spin polarization $P$ can be deduced for the materials used for the point contacts based on the greatest observed magnetoresistance, which we assume is due to magnetization processes like the ones illustrated in Fig. 2. $P$ is deduced from the Julière formula

$$
-\Delta \rho / \rho=2 P^{2} /\left(1+P^{2}\right) \text {. }
$$

The values are listed in Table I.

TABLE I. Point-contact magnetoresistance, spin polarization, and magnetostriction.

\begin{tabular}{lccc}
\hline \hline & $|\Delta \rho / \rho|_{\text {max }}(\%)$ & $P(\%)$ & $\lambda_{s}\left(10^{-6}\right)$ \\
\hline $\mathrm{Fe}_{3} \mathrm{O}_{4}$ & 94 & 94 & $19^{\mathrm{a}}$ \\
$\left(\mathrm{La}_{0.7} \mathrm{Sr}_{0.3}\right) \mathrm{MnO}_{3}$ & 43 & 52 & $13^{\mathrm{b}}$ \\
$\mathrm{Co}_{2} \mathrm{Cr}_{0.6} \mathrm{Fe}_{0.4} \mathrm{Al}$ & 81 & 83 & 5 \\
$\mathrm{MnBi}$ & 87 & 88 & $c$ \\
\hline \hline
\end{tabular}

${ }^{\mathrm{a}}$ See Ref. 13 .

${ }^{\mathrm{b}}$ See Ref. 14

${ }^{c}$ Unsaturated in $6 \mathrm{~T}$, where $\lambda_{s}=-200 \times 10^{-6}$.

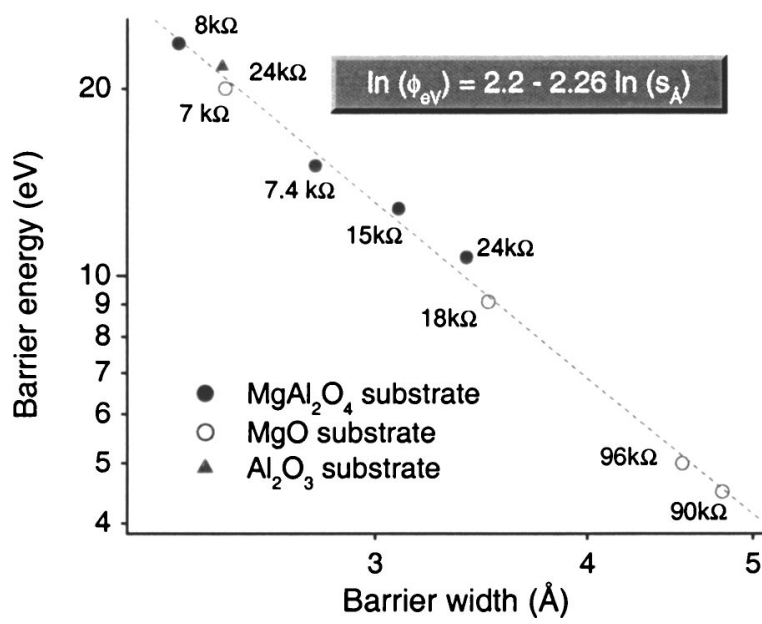

FIG. 5. Plot in a $\log -\log$ scale of the barrier height and width of nanoconstrictions obtained from the Simmons equation. The barrier height increases as the barrier width is reduced, as it happened with magnetite point contacts.

Another method of measuring point-contact magnetoresistance is in powder compacts. ${ }^{15}$ The measurement is simple, but the interpretation is not, as there are many conduction paths and the resistance is dominated by the least resistive contacts, which exhibit little magnetoresistance. A solution is to dilute the conducting powder with a nonconducting powder, and measure powder magnetoresistance near the percolation threshold. In that case the maximum magnetoresistance will be $-\Delta \rho / \rho \approx P^{2} /\left(1+P^{2}\right)$.

\section{B. Nanoconstrictions}

In order to avoid problems related to the short-lived stability of the point contacts, we studied a series of structures engraved in thin films by focused-ion beam (FIB) patterning using the $\mathrm{Ga}^{+}$ion beam in a dual-beam FEI Strata 235 instrument. We restrict our attention here to magnetite films, which were prepared by sputtering on $\mathrm{MgO}, \mathrm{Al}_{2} \mathrm{O}_{3}$, or $\mathrm{MgAl}_{2} \mathrm{O}_{4}$ substrates. Most of the films show the Verwey transition at 110-120 K. Single or double (peanut) constrictions were fabricated, and the magnetoresistance measured using a four-point method. Chemical analysis by energy dispersion in x-ray analysis of the FIB patterned nanostructures gave gallium contents of less than $1 \%$.

The behavior was again quite variable. The nonlinearity of the $I: V$ curve was correlated with $G$, and the $I-V$ characteristics were again fitted to Eq. (1). The two parameters $G$ and $C$ are related to the barrier height and width in the Simmons equation (Fig. 5). In this section we present the characteristics of contacts with $0.05 G_{0}<G<2 G_{0}$. The higherresistance contacts are described in the following section. Lower resistance contacts show linear $I: V$ curves and magnetoresistance below $2 \%$.

A significant change in resistance of the nanoconstriction is sometimes observed on applying a magnetic field. The applied field these experiments is $400 \mathrm{mT}$, but magnetostriction is excluded by the rigidity of the substrate. On applying the field to a nanoconstriction, the conductance can increase dramatically, varying from a high resistance state characteristic of a nanogap to a resistance in the nanoconstriction 


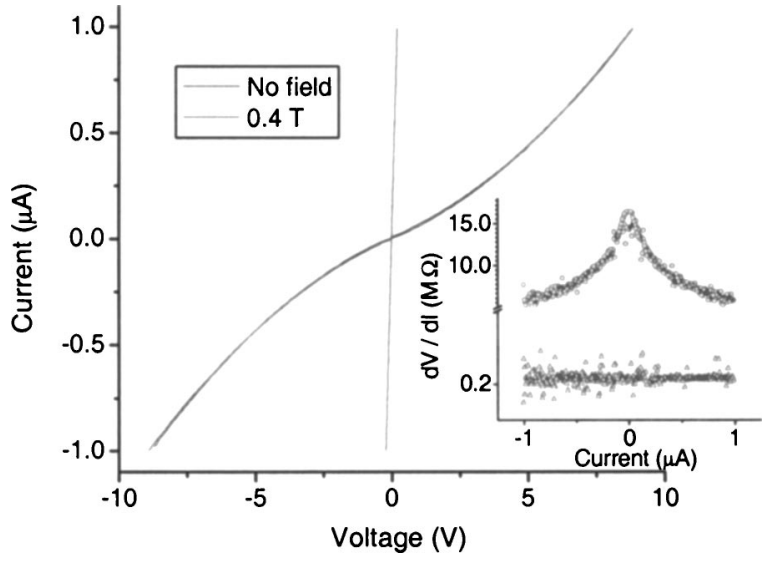

FIG. 6. $I-V$ and differential resistance for a magnetite nanoconstriction with an initial nonlinear $15 \mathrm{M} \Omega$ characteristic that turns into a linear $200 \mathrm{k} \Omega$ characteristic in the presence of a magnetic field of $400 \mathrm{mT}$.

range (Fig. 6). However, the constriction does not immediately revert to the high-resistance state on removing the field. This is a slow and gradual process, which may take a week or more to complete. When something like the initial highresistance state is restored, it can be switched back again to the low-resistance state by applying the field. It seems that the larger the magnetoresistance, the longer the recovery time. To speed up the recovery process, we applied a high current $(\sim 500 \mu \mathrm{A})$, which will form a different highresistance contact due to migration of the atoms in the vicinity of the nanoconstriction (Fig. 7). Similar magnetoresistance may be observed for this contact.

\section{Nanogap}

We consider a magnetite nanozone with a resistance of more than about $200 \mathrm{k} \Omega$ to be a nanogap. A different $I: V$ curve is found in this region, where the tunneling characteristic of Eq. (1), gives way to a steeper increase of current with voltage above a threshold. In these devices where the potential drop across the nanozone gives electric fields of order $10^{9} \mathrm{~V} \mathrm{~m}^{-1}$ or $1 \mathrm{~V} \mathrm{~nm}^{-1}$, the tunnel barrier is distorted to a triangular shape leading to field emission. ${ }^{16}$ The emitted electrons are accelerated across the nanogap, but they do not create a discharge there because the mean free path of the electrons in air is much longer than the width of the gap. The field-emission region is described by the Fowler-Nordheim equation $^{16}$

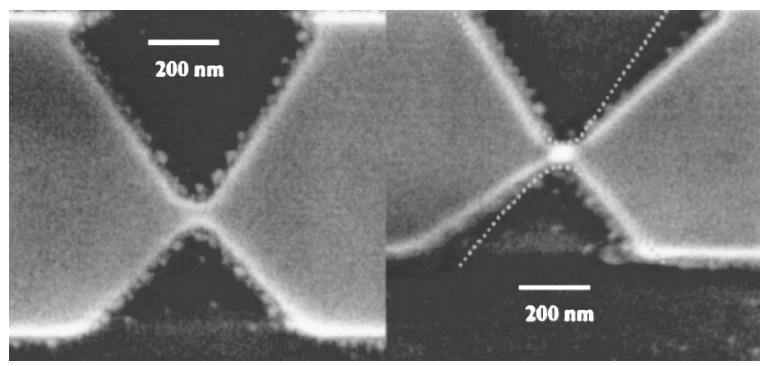

FIG. 7. Scanning electron microscopy images of a nanoconstriction before and after applying a high current of $500 \mu \mathrm{A}$.

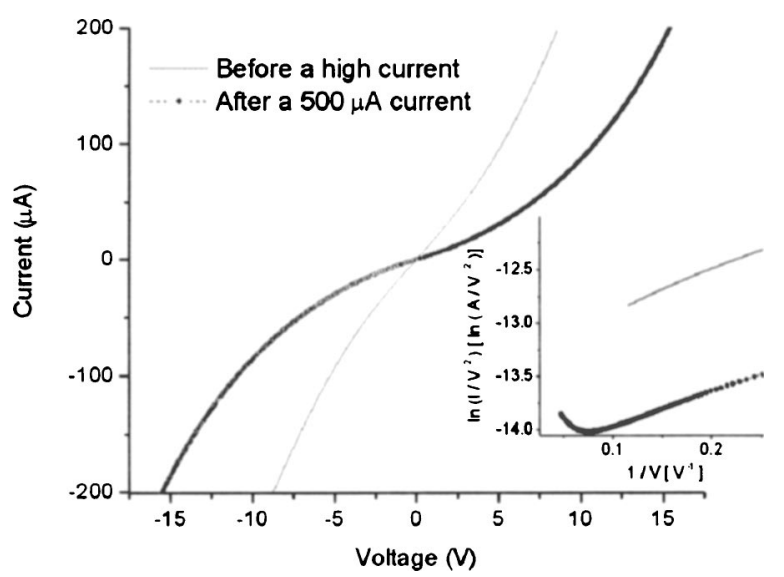

FIG. 8. $I: V$ and Fowler-Nordheim $(\mathrm{F}-\mathrm{N})$ plots of a sample before and after applying the current. The initial transport presents a maximum resistance of $60 \mathrm{k} \Omega$ and a positive F-N slope. After applying the current, the sample changes into a nanogap with a resistance of $250 \mathrm{k} \Omega$ and negative initial slope for the F-N plot.

$$
I=\left(a V^{2} / w^{2}\right) \exp \left(-b \psi^{3 / 2} w / V\right),
$$

where $a$ and $b$ are material constants, $\psi$ is the work function, and $w$ is the gap width. A plot of $\ln \left(I / V^{2}\right)$ vs $V^{-1}$ at high voltages has negative slope in the field-emission regime.

This field-emission regime can be induced by applying a high current $(>500 \mu \mathrm{A})$ into a low-resistance device. The current may displace material out of the constriction, changing the nanoconstriction into a nanogap, or modify the geometry in the vicinity of the nanostructure. In this way, by applying a high current across a nanoconstriction, we can observe an upturn in the Fowler-Nordheim plot that was not there before (Fig. 8).

We find that the slope of the Fowler-Nordheim plot near the onset of the field emission regime is dependent on the applied magnetic field, when there is no field dependence in the tunneling regime (Fig. 9). The magnetoresistance is positive, unlike the behavior reported for a magnetic semiconductor ${ }^{17}$ and there are variations of a few percent per tesla [Fig. 10(a)]. The dependence of the current near the inflexion point in the plot with the magnetic field can be

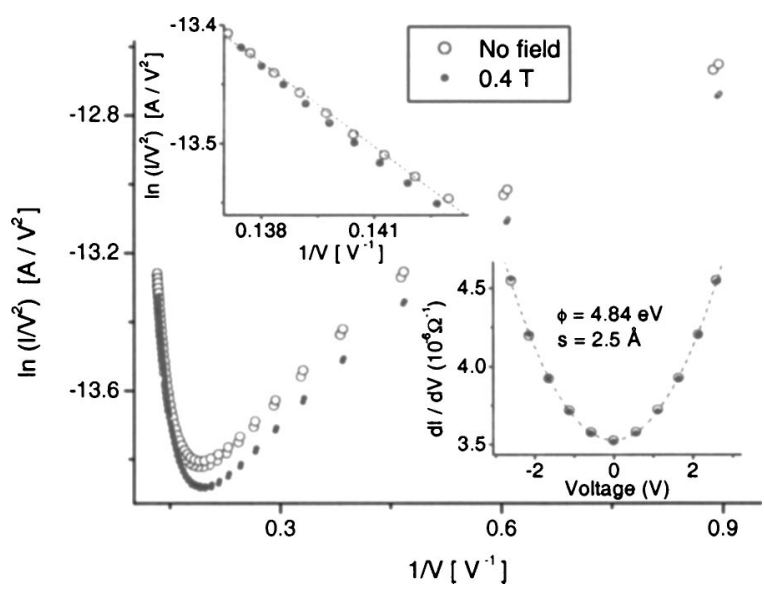

FIG. 9. F-N and tunneling in a nanogap at 0 and $0.4 \mathrm{~T}$. The tunneling parameters are the same with or without field, but the slope of the F-N plot in the field emission regime is increased in the presence of the magnetic field. 

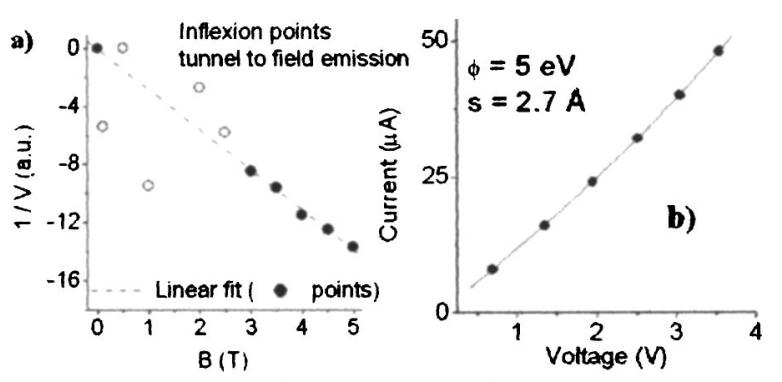

c)

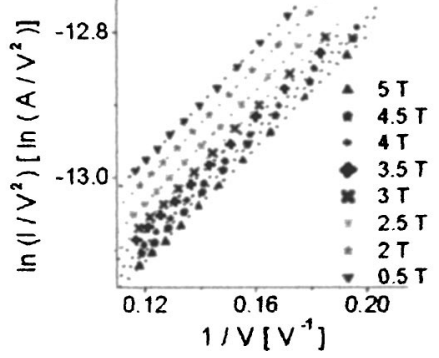

FIG. 10. (a) Slope in the F-N plot close to the onset of field emission at different magnetic fields. (b) Threshold voltage for field emission at the different magnetic fields. (c) Energy barrier of the device calculated from the Simmons fit.

analyzed [Fig. 10(b)] and the change of energy level can be deduced from the height of the barrier [Fig. 10(c)]. From this analysis we see a change of order $100 \mathrm{mV} \mathrm{T}^{-1}$ of the voltage at which field emission starts.

\section{DISCUSSION}

The conduction process in magnetite is hopping of small polarons, not diffusive spin transport as in a normal metal like $\mathrm{Au}, \mathrm{Ni}$, or $\left(\mathrm{La}_{0.7} \mathrm{Sr}_{0.3}\right) \mathrm{MnO}_{3}$. The difference in conduction process is evident in break junctions, where a series of conduction plateaus is observed for the metallic conductors, but not in magnetite where a histogram plot of resistivity shows a single weak peak at $G_{0}{ }^{10}$ The critical hop in a point contact across the interface from one surface to the next will be influenced by surface phonons as well as by an ill-defined adsorbed surface layer.

The first issue to consider is whether the point-contact magnetoresistance arises from mechanical or magnetic changes of the junction. If the two crystallites are magnetically decoupled and the only change is their relative magnetization, the transmission probability will have a prefactor $\cos ^{2} \theta_{i f} / 2$, which depends only on the relative orientation $\theta_{i f}=\left(\theta_{i}-\theta_{f}\right)$ of the magnetization on the initial and final sites of the hopping electron. The ratio $C / G$ should be the same for the in-field and zero-field states since it is determined by the nature of the junction. The other extreme is to suppose no direct local magnetic effect on the transmission probability, but only a magnetomechanical effect on the barrier. It is then expected that there will be a decrease in $C / G$ with field which follows the general trend displayed in Fig. 4. The truth seems to lie between these extremes: Fig. 11 shows the ratio $[C(B) / G(B)] /[C(0) / G(0)]$ plotted versus $G$. The ratio dips below 1 for the more resistive single-crystal contacts, but there is no such trend for the gold-coated or polycrystalline

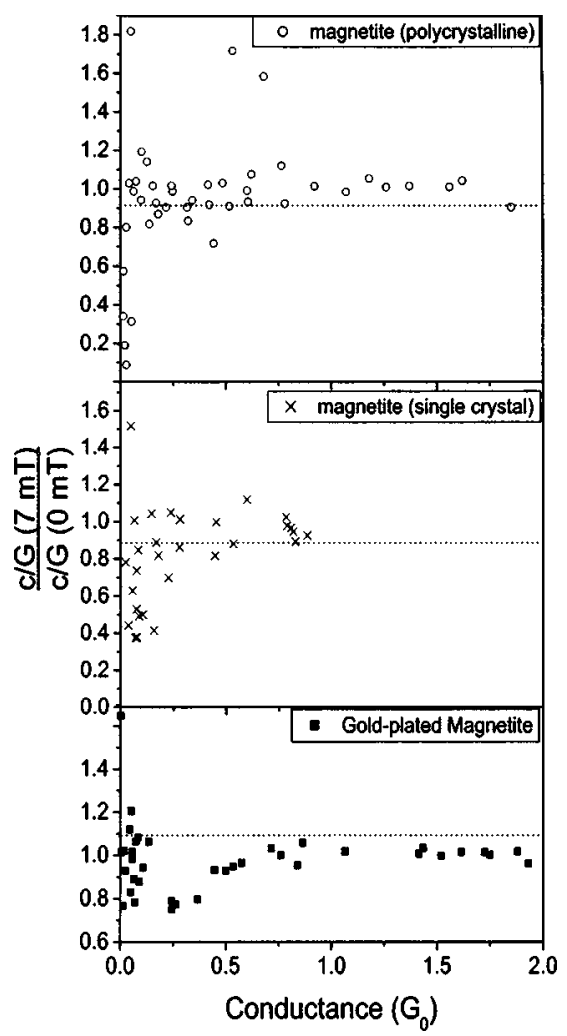

FIG. 11. Plot of the ratio $[C(B) / G(B)] /[C(0) / G(0)]$ showing the change of curvature of the $I: V$ plot when the magnetic field is applied. No change (a ratio of 1) indicates no mechanical change in the contact.

samples, suggesting that most of the magnetoresistance (MR) there is related to the spin-polarized transmission probability.

The role of magnetostriction in small fields was already discussed in Sec. II A. Another possible magnetomechanical effect arises from the dipole-dipole interaction between the crystallites. ${ }^{14}$ The dipolar force between the two crystallites is of order $\mu_{0} H_{d} M d^{2}$ where $d$ is the crystallite diameter and the field $H_{d}$ produced by one on the other is approximately $(1 / 4 \pi) M$. The strain $\varepsilon$ produced by this force is $\mu_{0} M^{2} / 4 \pi Y$, where $Y$ is Young's modulus. Taking $Y$ as $2 \times 10^{11} \mathrm{~N} \mathrm{~m}^{-2}$, and $M=400 \mathrm{kA} \mathrm{m}^{-1}$, the saturation magnetization for magnetite, gives $\varepsilon=8 \times 10^{-8}$. Since $d \approx 10^{-4} \mathrm{~m}$ for our magnetite crystallites, the change in size of the gap due to dipolar forces is at most $0.01 \mathrm{~nm}$, and therefore negligible. However, this would no longer be the case if $\mathrm{Ni}$ nanowires a millimeter long were used.

In the more conducting point contacts with no gold plating it is expected that exchange coupling propagates across the contact (gold acts as an exchange barrier). A domain wall is likely to form there, and another source of nonlinearity in the $I: V$ characteristic is spin pressure on the domain wall, ${ }^{10,18}$ which tends to displace it from the equilibrium position. The wall is also modified by the applied field, but in point contacts, the field reversibility indicates that if there is a domain wall at the contact, it returns to its original position when the field is removed.

The situation in our nanoconstrictions is different. While it is possible to observe large field-induced decreases of resistance, reversion to the original state is a very slow process. 
Mechanical changes should be eliminated by contact with the insulating substrate, the MR is independent of the direction of the applied field and the relaxation times are of the order of days. These points are not characteristic of a magnetostrictive process, so the magnetoresistance is probably due to a change in magnetic configuration of the nanoconstriction, perhaps by the elimination of a domain wall there. We have no method of determining the spin configuration in the nanoconstriction, but it may differ from the bulk because the ferromagnetic $B-B$ interaction in magnetite depends on the hopping electron, whereas the $A-A$ and $A-B$ superexchange interactions are negative. ${ }^{19}$ Once the wall is eliminated from the nanoconstriction, it is a slow process to renucleate it. The energy involved in the wall itself is $A \delta$, $10^{-19}-10^{-20} \mathrm{~J}$, but the energy $\Delta$ needed to nucleate it must be a little greater since the time $\tau$ required is of order $\tau_{0} \exp (\Delta / k T)$ where $\tau_{0}^{-1}$ is an attempt frequency of order $10^{10} \mathrm{~s}^{-1}$. The process is akin to magnetization reversal far from the coercive field in a bulk ferromagnet.

The changeover from a nanoconstriction to a nanogap by passing a current indicates electromigration, some direct evidence of which may be seen in Fig. 7(a). The contact can be created or blown away by the current. The behavior of the nanogap is in a magnetic field is puzzling. The $10 \mathrm{meV} \mathrm{T}^{-1}$ field effect in the field-emission region corresponds to a positive magnetoresistance which is difficult to explain in terms of a field effect on the barrier height. The chemical potential, defined by the $B$-site $\downarrow$ electrons at the Fermi level, is raised by the field, so the work function and barrier height should decrease. Even making allowance for the unrealistic barrier heights given by the Simmons fits, an increase of $10 \mathrm{meV} \mathrm{T}^{-1}$ is implausible, since it would imply $g$ factors of order 1000. It seems more plausible to associate the changes in the barrier height with the variation of the exchange energy as the electrodes at both sides of the nanogap align their magnetization with the field. This variation could easily be of order $10 \mathrm{meV} \mathrm{T}^{-1}$. This explanation would be further confirmed by the dependence of the barrier height with the barrier width found both in magnetite point contacts and nanoconstrictions, but not in Au-covered magnetite point contacts (see Figs. 4 and 5). As the electrodes approach each other, the exchange energy increases, and so does the barrier height. When the electrodes are covered with gold $(10 \mathrm{~nm}$ thick), there is no exchange until the electrodes are very near or touching ( $\rightarrow$ forming a nanocontact), therefore the barrier height remains constant.

\section{CONCLUSIONS}

Magnetic field effects have been found on the resistance of magnetite nanoconstrictions and nanogaps, and extensive data presented on nanocontacts. There is good evidence that the large effects found in the more resistive structures $(G$ $<G_{0}$ ) cannot be explained by magnetically induced strain, ${ }^{20}$ but that they reflect the spin-dependent hopping or tunneling probability of the electron across the contact. The data are compatible with a high degree of spin polarization of magnetite at room temperature.

Interpretation of the results in terms of domain-wall motion remains speculative in the absence of precise structural and magnetic characterization of the nanozone. We cannot tell, for example, whether a nanogap is actually a tiny air gap, or a zone of insulating Ga-containing oxide at the surface. Electromigration can be a significant factor in these structures, given the electric fields and current densities present at the contact $\left(\approx 10^{9} \mathrm{~V} \mathrm{~m}^{-1}\right.$ and $\left.\approx 10^{12} \mathrm{~A} \mathrm{~m}^{-2}\right)$. Reversible, field or current-controllable switching in a tailored nanoconstriction, the peanut device for example, ${ }^{9,21}$ remains a tantalizing prospect.

\section{ACKNOWLEDGMENTS}

This work was supported by Enterprise Ireland and Science Foundation Ireland. The authors are grateful to Marin Gospodinov, William Egglehof, and Michael Ziese for providing some of the samples.

${ }^{1}$ N. Garcia, M. Muñoz, and Y.-W. Zhao, Phys. Rev. Lett. 82, 2923 (1999).

${ }^{2}$ N. Garcia et al., J. Magn. Magn. Mater. 272, 1722 (2004).

${ }^{3}$ M. Viret et al., Phys. Rev. B 66, 220401 (2002).

${ }^{4}$ R. A. Buhrman, O. Ozatay, P. Chalsani, N. C. Emley, and I. Krivorotov, J. Appl. Phys. 95, 7315 (2004).

${ }^{5}$ P. Bruno, Phys. Rev. Lett. 83, 2425 (1999).

${ }^{6}$ Y. Labaye, L. Berger, and J. M. D. Coey, J. Appl. Phys. 91, 5341 (2002).

${ }^{7}$ J. M. D. Coey, in Spin Electronics, edited by M. Ziese and M. Thornton

(Springer, Berlin, 2001), Chap. 12; J. M. D. Coey and S. Sanvito, J. Phys. D 37, 988 (2004).

${ }^{8}$ J. J. Versluijs, Ph.D. thesis, Trinity College, Dublin, 2002.

${ }^{9}$ J. J. Versluijs, M. A. Bari, and J. M. D. Coey, Phys. Rev. Lett. 87, 026601 (2001).

${ }^{10}$ J. M. D. Coey, J. J. Versluijs, and M. Venkatesen, J. Phys. D 35, 2457 (2002).

${ }^{11}$ E. Clifford, M. Venkatesan, and J. M. D. Coey, J. Magn. Magn. Mater. 272-276, 1614 (2004).

${ }^{12}$ J. G. Simmons, J. Appl. Phys. 34, 1793 (1963).

${ }^{13}$ H. Bourdonnay et al., J. Phys. (Paris), Colloq. 32, C1-1182 (1971).

${ }^{14} \mathrm{~W}$. F. Egelhoff et al. (unpublished).

${ }^{15}$ J. M. D. Coey, J. Appl. Phys. 85, 5576 (1999).

${ }^{16}$ R. H. Fowler and L. Nordheim, Proc. R. Soc. London, Ser. A 119, 173 (1928).

${ }^{17}$ T. Siegrist, S. von Molnar, and F. Holtzberg, Appl. Phys. Lett. 47, 1087 (1985).

${ }^{18}$ G. Tatara and H. Kohno, Phys. Rev. Lett. 92, 086601 (2004).

${ }^{19}$ C. P. Hunt, B. M. Moskowitz, and S. K. Bannerjee, in Rock Physics and Phase Relations, Handbook of Physical Constants, edited by J. Ahrens (AGU, Washington, 1995)

${ }^{20}$ R. V. Demin, L. I. Koroleva, and A. M. Balbashov, J. Magn. Magn. Mater. 177-181, 871 (1998).

${ }^{21}$ C. Rüster et al., Phys. Rev. Lett. 91, 216602 (2003). 